\title{
Boundary Processing of HHT Using Support Vector Regression Machines
}

\author{
Wu Wang, Xueyao Li, and Rubo Zhang \\ National Laboratory on Machine Perception, Peking University, Beijing, P.R. China \\ College of Computer Science and Technology, Harbin Engineering University, \\ Harbin150001, China \\ wangwu131@163.com
}

\begin{abstract}
In order to better restrain the end effects in Hilbert-Huang Transform, support vector regression machines (SVRM), which have the superiority in the time series prediction, are adopted to extend the data at the both ends. In the application of SVRM, the parameters have a great influence on the performance of generalization. In this paper the influence of parameters is discussed, and then an adaptive support vector regression machine is proposed based on the particle swarm optimization (PSO) algorithm. With the parameters optimized by PSO, SVRM can be characterized as self-adaptive and high generalization performance in applications. Experiments show that this method can solve the problem of selecting parameters properly. Contrast to the neural networks methods and HHTDPS designed by Huang et al., end effects can be restrained better and the Intrinsic Mode Functions have less distortion.
\end{abstract}

Keywords: End effects, Hilbert-Huang Transform, Support vector regression machines, Particle swarm optimization.

\section{Introduction}

Hilbert-Huang Transform (HHT) is a powerful data analysis method for nonlinear and non-stationary data[1][2], which is based on empirical mode decomposition (EMD). Although Hilbert-Huang transform is a powerful analysis method for non-stationary signals, but end effects are its unavoidable problem[1][4][5]. If the end effects aren't restrained effectively, it can produce large swings and eventually propagate inward and corrupt the whole data[1]. So it is necessary to restrain the end effects effectively. Many new methods have proposed in recent years, such as time series prediction based on neural networks[3], mirror extending method[4], waveform matching method[5], constructing two periodic series from the original data by even and odd extension[6]. Among these methods, the neural networks have relatively better effects than other methods[7]. The neural networks extending method can work well for non-stationary signals[3], but the local minimum point, over learning and the excessive dependence on experience about the choice of structures and types are its inevitable limitation. A potential solution to the above problems is to use Support vector regression machines (SVRM). In applications, the performance of SVRM is sensitive to its parameters[8], so in this paper the particle swarm optimization (PSO) algorithm is proposed to optimize the parameters. More importantly, with the parameters optimized by PSO, this method can be self-adaptive to nonlinear and non-stationary data. 


\section{Parameter Selection in SVRM Based on PSO}

In the application of Support vector regression machines, there are three important parameters: the regularization parameter $C$, the tube size $\mathcal{E}$ and the kernel parameter $\sigma^{2}$. All of them have a great influence on the generalization performance of the SVRM. In this paper, PSO is proposed to optimize these parameters. Compared to other parameter selection methods, this approach is simple but faster[9]. In PSO, the potential solutions, called particles, fly through the problem space with a velocity, which is dynamically adjusted according to its own flying experience and its companions' flying experience. Every particle is evaluated by the fitness value. The best previous position of the particle is recorded and represented as pbest. The position of the best particle among all the particles in the population is represented by the symbol gbest. According to the pbest and gbest, the particle updates its velocity and position as the following equation:

$$
\begin{aligned}
& v_{i d}=w^{*} v_{i d}+c_{1} * \operatorname{rand}() *\left(\text { pbest }_{i d}-x_{i d}\right)+c_{2} * \operatorname{Rand}() *\left(\text { gbest }-x_{i d}\right) \\
& x_{i d}=x_{i d}+v_{i d}, i=1,2, \cdots M
\end{aligned}
$$

where $d$ is the dimension of every particle, which is equivalent to the number of parameters to optimize. Rand ( ) and the Rand ( ) are two random numbers generated independently. $w$ is inertia weight, $c_{1}$ and $c_{2}$ are learning factors and the $M$ is the population size.

To get better generalization ability, it is necessary to make the SVRM model have lower error rate and a simple structure. Therefore, the fitness function can be defined as follows:

$$
F_{i}=f_{\text {error }}+K \times \frac{N_{s v}}{N} \times\left(1-f_{\text {error }}\right)
$$

where $f_{\text {error }}$ is the error rate in the training data sets, $N_{s v}$ is the number of the support vectors $(\mathrm{SV})$ and $N$ is the training data number. $K \in[0,1]$ is used to make a balance between the precision and the complexity.

\section{Implementation and Experimental Results}

In order to make a comparison between the SVRM and RBF network in forecasting effect, one speech segment of 731 data points and the $S N R=5 \mathrm{~dB}$ was picked as the training samples, which is shown in Fig.1. The data were forecast 150 data points from the back endpoint purposely, but in applications, we only need to forecast very less points. The results by SVRM and RBF neural network are shown in Fig. 2 and Fig. 3, where the dashed line represents the signal forecast by SVRM or RBF network and the solid line represents the actual signal. Figures show the forecasted series by RBF network have a more serious distortion. The mean absolute percentage error (MAPE) of the SVRM and RBF network were 1.1012 and 1.3470. The result also demonstrates that the forecasting method based on SVRM is superior to that based on neural network. 


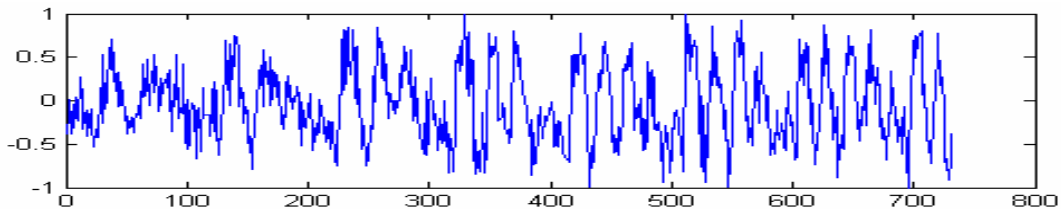

Fig. 1. The noised speech signal with $\mathrm{SNR}=5 \mathrm{~dB}$

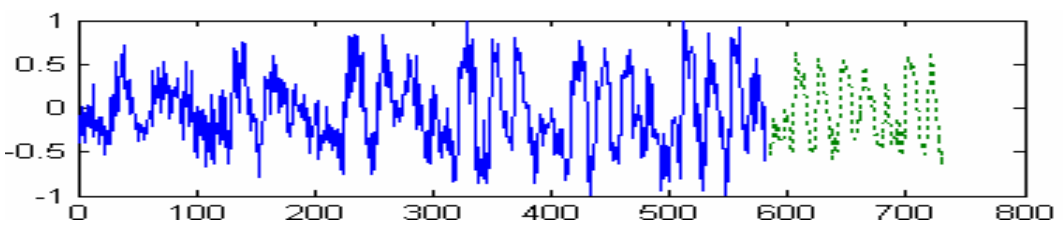

Fig. 2. The noised speech signal after the SVRM predicting

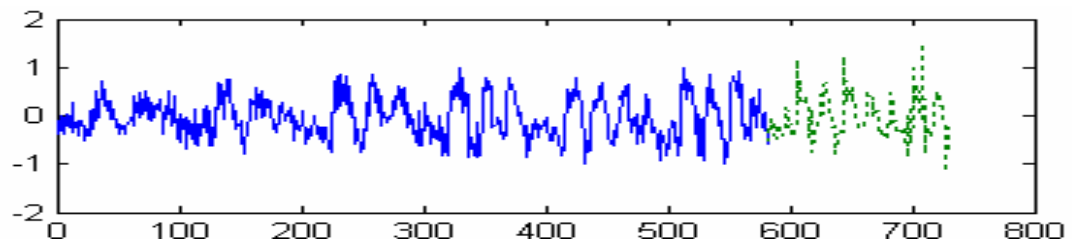

Fig. 3. The noised speech signal after the RBF neural network predicting
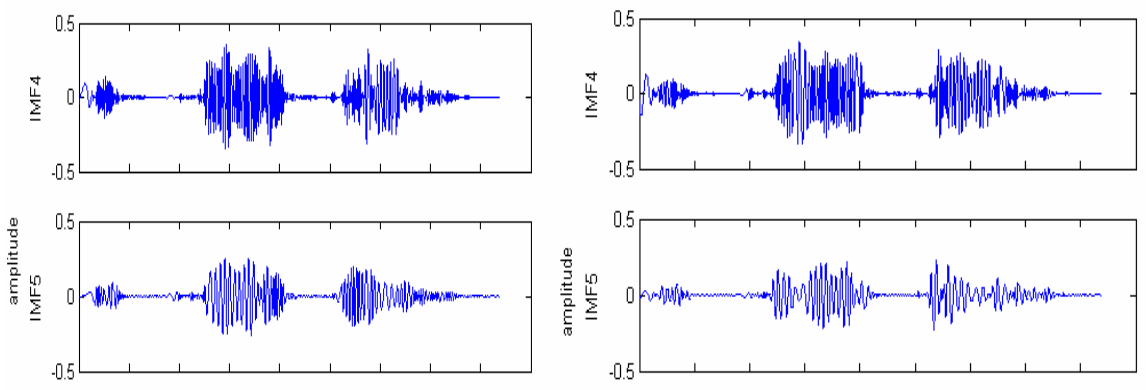

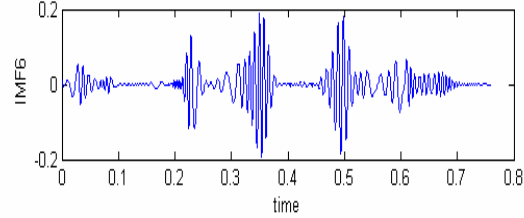

(a)

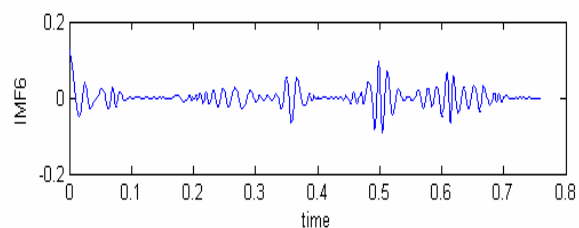

(b)

Fig. 4. The EMD decomposition results of the speech signal after being forecast by the SVRM (a) and the HHTDPS (b) 
We also conducted our experiments on a speech material of 0.7608 s long. Fig.4 shows the IMFs results extracted form our method and decomposed by the HHT Data Processing System (HHTDPS). The HHTDPS is an engineering spectral analysis tool developed at Goddard Space Flight Center (GSFC) of NASA. The HHTDPS used is Version 1.4, which is the latest version of HHT software.

Fig. 4 shows the IMF components after forecasting by SVR and got by HHTDPS respectively. From the Fig.4 (b) we can see the fourth and sixth IMF components in HHTDPS have an explicit distortion at the starting point, while this distortion can be restrained effectively by our algorithm, shown in Fig.4(a).

\section{Conclusion}

In this paper, we proposed a method to restrain the end effects in HHT by time series prediction based on adaptive support vector regression machine. For the end effects in Hilbert-Huang transform, the original time series can be forecast by SVRM, and the end effects can be released by abandoning the extended data. With the parameters optimized by PSO, SVRM can be self-adaptive to nonlinear and non-stationary data. It is helpful for SVRM to get high generalization performance in time series prediction. Therefore, SVRM is a very suitable forecasting method for the time series. Our experiments show end effects in HHT can be restrained effectively by this method.

Acknowledgments. This work is supported by the NSFC No. 60475016 and the Foundational Research Fund of Harbin Engineering University HEUF04092. We also appreciate NASA Goddard to approve us to use and evaluate HHTDPS software.

\section{References}

1. Norden E. Huang .The Empirical Mode Decomposition and the Hilbert spectrum for nonlinear and non-stationary time series analysis J. proc.R.Soc. Lond.A (1998) 454,903-995.

2. Norden E.Huang. A confidence limit for the Empirical Mode Decomposition and Hilbert spectral analysis; J.proc.R.Soc.Lond 2003(459), 2317-2345.

3. Y.J. Deng, W. Wang, Boundary processing technique in EMD method and Hilbert transform, Chinese Science Bulletin 46 (11) (2001) 257-263.

4. J.P. Zhao, D.J. Huang, Mirror extending and circular spline function for empirical mode decomposition method, Journal of Zhejiang University(Science),2001, 2(3) 247-252

5. Q. Gai, X.J. Mao, H.Y. Zhang, et al., New method for processing end effect in local wave method, Journal of Dalin University of Technology, Chinese 42 (1) (2002) 115-117.

6. K. Zeng and M.X. He, A Simple Boundary Process Technique for Empirical Mode Decomposition, IGARSS '04. Proceedings, 6(2004) 4258 - 4261.

7. B.J. Xu, J.M. Zhang, X.L. Xu, J.W. Li, A study on the method of restraining the ending effect of empirical mode decomposition, Transactions of Beijing Institute of Technology, 26(3)(2006) 196-200.

8. C. C. Chuang, S. F. Su, J. T. Jeng, and C. C. Hsiao, Robust support vector regression networks for function approximation with outliers, IEEE Trans. Neural Netw., vol. 13, no. 6, pp. 1322-1330, Nov. 2002.

9. Kennedy, I. and Eberhan, R. C. Paticle swarm optimization. Proceedings of IEEE Inlemational Conference on Neural Networks. Piscalaway, NJ. pp. 1942-1948, 1995. 